\title{
Biomechanical factors and physical examination findings in osteoarthritis of the knee: associations with tissue abnormalities assessed by conventional radiography and high-resolution 3.0 Tesla magnetic resonance imaging
}

Jesper Knoop ${ }^{1 *}$, Joost Dekker ${ }^{2,3}$, Jan-Paul Klein ${ }^{4}$, Marike van der Leeden ${ }^{1,2}$, Martin van der Esch ${ }^{1}$, Dick Reiding ${ }^{5}$, Ramon E Voorneman ${ }^{5}$, Martijn Gerritsen ${ }^{5}$, Leo D Roorda ${ }^{1}$, Martijn PM Steultjens ${ }^{6}$ and Willem F Lems ${ }^{5,7}$

\begin{abstract}
Introduction: We aimed to explore the associations between knee osteoarthritis (OA)-related tissue abnormalities assessed by conventional radiography (CR) and by high-resolution 3.0 Tesla magnetic resonance imaging (MRI), as well as biomechanical factors and findings from physical examination in patients with knee OA.

Methods: This was an explorative cross-sectional study of 105 patients with knee OA. Index knees were imaged using CR and MRI. Multiple features from CR and MRI (cartilage, osteophytes, bone marrow lesions, effusion and synovitis) were related to biomechanical factors (quadriceps and hamstrings muscle strength, proprioceptive accuracy and varus-valgus laxity) and physical examination findings (bony tenderness, crepitus, bony enlargement and palpable warmth), using multivariable regression analyses.

Results: Quadriceps weakness was associated with cartilage integrity, effusion, synovitis (all detected by MRI) and CR-detected joint space narrowing. Knee joint laxity was associated with MRI-detected cartilage integrity, CRdetected joint space narrowing and osteophyte formation. Multiple tissue abnormalities including cartilage integrity, osteophytes and effusion, but only those detected by MRI, were found to be associated with physical examination findings such as crepitus.

Conclusion: We observed clinically relevant findings, including a significant association between quadriceps weakness and both effusion and synovitis, detected by MRI. Inflammation was detected in over one-third of the participants, emphasizing the inflammatory component of $\mathrm{OA}$ and a possible important role for anti-inflammatory therapies in knee OA. In general, OA-related tissue abnormalities of the knee, even those detected by MRI, were found to be discordant with biomechanical and physical examination features.
\end{abstract}

\section{Introduction}

Osteoarthritis (OA) of the knee involves many tissues of the knee joint, not only addressing cartilage but also including abnormalities in subchondral bone and the synovial membrane $[1,2]$. Most people with knee OA suffer from pain, stiffness and limitations in daily activities

\footnotetext{
* Correspondence: j.knoop@reade.nl

'Amsterdam Rehabilitation Research Center - Reade, Dr. Jan van

Breemenstraat 2, 1056 AB Amsterdam, the Netherlands

Full list of author information is available at the end of the article
}

[2]. Physical examination may reveal clinical signs such as joint crepitus, swelling, deformities or increased warmth of the joint [2]. Additionally, biomechanical factors such as lower limb muscle strength, proprioceptive accuracy of the knee joint and varus-valgus knee joint laxity, which are considered essential factors for knee stabilization [3-5], have frequently been found to be impaired in knee OA patients [6-8]. Besides being clinically important consequences of $\mathrm{OA}$, biomechanical factors may also play a role in the onset of tissue abnormalities [9-12]. Presumably, 
biomechanical factors in the knee joint and tissue abnormalities interact with each other during the disease process of OA.

Conventional radiography (CR) is the primary modality for disease diagnosis and classification in clinical practice [13]. CR-based joint space width (JSW), an indirect measure for cartilage loss, is the most important outcome measure in pharmacological studies [14]. In contrast to radiography, magnetic resonance imaging (MRI) is able to visualize cartilage and to detect bone marrow lesions (BML) and inflammation (for example, effusion and synovitis) [14]. MRI is therefore currently the best modality available for imaging OA-related tissue abnormalities [14] and may be able to unravel mechanisms underlying biomechanical impairments.

Only a few studies have been performed on the association between tissue abnormalities and biomechanical factors. While studies using CR provided mixed results [15-19], studies using MRI clearly demonstrated an association between (medial tibiofemoral and patellafemoral compartmental) cartilage thickness and quadriceps strength $[18,20,21]$. A small number of studies (using CR or MRI) provided mixed results on the relationship between tissue abnormalities (namely, cartilage thickness $[8,22,23]$ and osteophyte formation $[22,24])$ and knee joint laxity, while proprioceptive accuracy and hamstrings muscles have never been studied in relation to tissue abnormalities.

Most studies concerning OA-related tissue abnormalities focused on the association with patient-reported pain or activity limitations, generally providing evidence for discordance - particularly in studies using CR [13,25-32]. OA-related tissue abnormalities are possibly more closely linked to findings from physical examination, rather than to self-reported outcomes. Recently, a population-based study using MRI showed that multiple tissue abnormalities were related to the presence of crepitus [33]. As far as we know, tissue abnormalities have not so far been related to findings from physical examination in a knee OA population.

In conclusion, there is limited knowledge on the association between OA-related tissue abnormalities, biomechanical factors and physical examination findings in knee OA. The first aim of the present study was therefore to explore associations of CR-detected and MRI-detected tissue abnormalities and biomechanical factors (quadriceps and hamstrings muscle strength, proprioceptive accuracy and knee joint laxity) in patients with knee OA. The second aim was to explore associations of CRdetected and MRI-detected tissue abnormalities and physical examination findings (bony tenderness, crepitus, bony enlargement and palpable warmth) in patients with knee OA.

\section{Materials and methods Subjects}

For the present study, participants were recruited from a randomized controlled trial on the effectiveness of a knee stabilization exercise program [34]. Inclusion criteria were clinical knee OA diagnosis according to the American College of Rheumatology criteria (that is, presence of knee pain and at least three of the following six items: age $>50$ years, morning stiffness $<30$ minutes, crepitus, bony tenderness, bony enlargement and no palpable warmth) [35], age between 40 and 75 years, instability of the knee and written informed consent. Total knee arthroplasty, rheumatoid arthritis or any other form of arthritis (that is, crystal arthropathy, septic arthritis, spondylarthropathy), comorbidities affecting daily functioning, severe knee pain (numeric rating scale $>8$ ) and/ or contraindication for MRI (for example, pacemaker, claustrophobia) were exclusion criteria. Patients were subsequently examined by radiologists, rheumatologists, and physiatrists. The measurement protocol contained assessment of demographic, biomechanical and clinical factors related to OA, as well as CR and MRI, all assessed prior to the start of the trial. All participants provided written informed consent. The study was approved by the Reade/Slotervaart Institutional Review Board.

\section{Index knee}

For knee-specific variables we used data from the index knee. For unilateral knee OA patients, the index knee was the knee that was diagnosed with clinical OA. For bilateral knee OA patients, the index knee was the knee that most severely affected daily activities on patient self-report.

\section{Biomechanical factors}

Measurements of lower limb muscle strength, proprioceptive accuracy and varus-valgus laxity have been extensively described in previous publications [5,36,37]. In summary, muscle strength was measured isokinetically $\left(60^{\circ} /\right.$ second) for both knee extension (quadriceps) and flexion (hamstrings) strength. Strength outcomes (in Nm) were adjusted for bodyweight [36]. For proprioceptive accuracy (knee joint motion sense), a threshold detection task was used - which assessed the amount of degrees after motion detection, with motion velocity of $0.3 \%$ second [36]. Varusvalgus knee joint laxity was measured as the total range of knee motion (in degrees) in the frontal plane. In a sitting position, the thigh and lower leg were fixed at five places to prevent for medial or lateral movement of the thigh and lower leg and for hip rotation. In a fixed knee flexion of $20^{\circ}$, a load of $1.12 \mathrm{~kg}(7.7 \mathrm{Nm})$ was applied to the lower leg both medially and laterally, resulting in varus or valgus movement across the transverse axis of the knee joint [37]. 


\section{Physical examination findings}

The following features from the American College of Rheumatology criteria for clinical knee OA diagnosis were assessed by the physiatrist on physical examination of the knee joint: bony tenderness (that is, pain by palpation at the joint line), crepitus (that is, crackling or grinding sound in the joint during weight bearing), bony enlargement at joint line and palpable warmth of the knee joint [35]. Findings were scored as yes (present) or no (absent).

\section{Radiography}

Conventional radiographs of tibiofemoral joints were made by a weight-bearing posterioanterior view, semiflexed $\left(7\right.$ to $\left.10^{\circ}\right)$ according to Buckland-Wright and colleagues [38]. Radiographs of patellofemoral joints were made by a single standing mediolateral view in $30^{\circ}$ flexion, and a skyline (inferior superior) view in $30^{\circ}$ flexion [39]. Two independent observers (DR, MvdE) graded radiographs, unaware of the patient's clinical characteristics. One observer (DR) was a bone and joint radiologist, and the second observer (MvdE) was an epidemiologist trained by two musculoskeletal radiologists. The JSW and osteophyte formation were scored on a scale of 0 to 3 , for medial tibiofemoral (MTF), lateral tibiofemoral (LTF) and patellafemoral (PF) compartments separately, according to the Osteoarthritis Research Society International (OARSI) atlas [40]. Severity of structural damage in the knee, according to Kellgren-Lawrence [41], was also scored. The intraclass correlation coefficient for interrater reliability in 64 knees was $0.87(P<0.001)$ for JSW, $0.60(P<0.001)$ for osteophytes and $0.89(P<0.001)$ for the Kellgren-Lawrence score.

\section{Magnetic resonance imaging}

MRI scans of one knee (the index knee) were performed by a 3.0 Tesla whole-body magnetic resonance scanner (General Electric Medical Systems, Milwaukee, WI, USA) using a phased array knee coil. The MRI examination included five scans. All scans were made with a field of view of $180 \mathrm{~mm}$. The first sequence was a sagittal proton density-weighted turbo spin-echo with fat suppression (slice thickness $3 \mathrm{~mm}$; interslice gap $0.3 \mathrm{~mm}$; repetition time 3,480 milliseconds; echo time 42 milliseconds; turbo factor 8 ; matrix $384 \times 256$ ). The second sequence was a sagittal T1-weighted turbo spin-echo (slice thickness $3 \mathrm{~mm}$; interslice gap $0.3 \mathrm{~mm}$; repetition time 760 milliseconds; echo time 14 milliseconds; turbofactor 2; matrix $384 \times 256$ ). The third sequence was a coronal T2-weighted turbo spin-echo with fat suppression (slice thickness $3 \mathrm{~mm}$; interslice gap $0.3 \mathrm{~mm}$; repetition time 5,800 milliseconds; echo time 85 milliseconds; turbo factor 15; matrix $384 \times 256)$. The fourth sequence was a sagittal combined multi-echo gradient echo (thickness $3.5 \mathrm{~mm}$; interslice gap
$0.3 \mathrm{~mm}$; repetition time 973 milliseconds; excitation angle $20^{\circ}$; matrix $352 \times 224$ ). The last sequence was a coronal combined multi-echo gradient echo (thickness $3.0 \mathrm{~mm}$; interslice gap $0.5 \mathrm{~mm}$; repetition time 854 milliseconds; excitation angle $20^{\circ}$; matrix $352 \times 224$ ).

MRI scans were assessed according to the BostonLeeds Osteoarthritis Knee Score system [42], a semiquantitative whole-joint scoring method. A radiologist (J-PK) with 27 years of musculoskeletal expertise, blinded to the patient's clinical characteristics and radiographic assessment, assessed all MRI scans. Cartilage integrity, osteophyte formation and BML were scored per region, with scores ranging from 0 (no abnormality) to 3 (severe abnormality). For effusion, one knee-specific score was used, ranging from 0 (physiological amount of effusion) to 3 (large effusion). Presence of synovitis (yes/no) was assessed in five regions separately. Specific details on MRI assessment are presented in Table 1 . The intraclass correlation coefficient for intrarater reliability in 15 knees was found to be $0.83(P<0.001)$ for cartilage thickness, $0.86(P<0.001)$ for osteophytes, $0.91(P<0.001)$ for BML, and $0.97(P<0.001)$ for effusion. Cohen's kappa for synovitis was $0.73(P=0.003)$.

\section{Statistical analysis}

Firstly, descriptive statistics were calculated. Secondly, linear and logistic regression analyses were performed for associations of tissue abnormalities (independent variables) with biomechanical factors and physical examination findings (dependent variables), for continuous and dichotomous scales respectively. Compartment-specific scores for JSW and osteophytes, detected by CR, were dichotomized by combining scores 0 and 1 (that is, only minute abnormality) and combining scores 2 and 3 (that is, at least definite abnormality) for each compartment separately. Region-specific scores (0 to 3 ) for cartilage integrity, osteophytes and BML, detected by MRI, were summed into compartment-specific scores [27,43-45]. Knee-specific scores for MRI-based effusion were dichotomized, by combining scores 0 and 1 (physiological amount/small effusion) and by combining scores 2 and 3 (medium/large effusion) [28,46]. Regionspecific scores for MRI-based synovitis were also dichotomized into one knee-specific score (no synovitis at all vs. synovitis present in at least one region). Regression analyses were performed univariably as well as multivariably with adjustment for age, gender and duration of knee symptoms. Standardized regression $\beta$ coefficients and $P$ values were estimated for linear regression analyses; odds ratios and $P$ values were estimated for logistic regression analyses. Statistical significance was accepted at $P<0.05$. All analyses were performed using PASW Statistics 18.0 (SPSS Inc., Chicago, IL, USA). 
Table 1 Magnetic resonance imaging assessment, according to Boston-Leeds Osteoarthritis Knee Score [42]

\begin{tabular}{|c|c|c|c|c|c|c|}
\hline & & Cartilage integrity & Osteophytes & Bone marrow lesions & Effusion & Synovitis \\
\hline Assessment & & $\begin{array}{l}\text { Size of any cartilage } \\
\text { thickness loss (both } \\
\text { partial and full thickness } \\
\text { loss) as percentage of } \\
\text { cartilage surface area of } \\
\text { each region }\end{array}$ & $\begin{array}{l}\text { Size of protuberance of } \\
\text { osteophyte }\end{array}$ & $\begin{array}{l}\text { Size of BML (including volume } \\
\text { of any associated cysts) as } \\
\text { percentage of bone volume of } \\
\text { each region }\end{array}$ & $\begin{array}{l}\text { Size of } \\
\text { effusion } \\
\text { within } \\
\text { synovial } \\
\text { space }\end{array}$ & $\begin{array}{l}\text { Presence of } \\
\text { synovitis }\end{array}$ \\
\hline \multirow[t]{17}{*}{ Region } & Number & 8 & 12 & 9 & $\begin{array}{l}1 \text { (one score } \\
\text { for whole } \\
\text { knee) }\end{array}$ & 5 \\
\hline & \multirow[t]{2}{*}{ MTF } & $\begin{array}{l}\text { Medial weight-bearing } \\
\text { femur }\end{array}$ & Medial weight-bearing femur & Medial weight-bearing femur & - & - \\
\hline & & Medial region tibia & $\begin{array}{l}\text { Medial posterior region femur } \\
\text { Medial region tibia }\end{array}$ & Medial region tibia & & \\
\hline & \multirow[t]{3}{*}{ LTF } & $\begin{array}{l}\text { Lateral weight-bearing } \\
\text { femur }\end{array}$ & Lateral weight-bearing femur & lateral weight-bearing Femur & - & - \\
\hline & & Lateral region tibia & Lateral posterior region femur & Lateral region tibia & & \\
\hline & & & Lateral region tibia & & & \\
\hline & \multirow[t]{6}{*}{ PF } & Medial region patella & Superior region patella & Medial region patella & - & - \\
\hline & & Lateral region patella & Inferior region patella & Lateral region patella & & \\
\hline & & Medial trochlea femur & Medial region patella & Medial trochlea femur & & \\
\hline & & Lateral trochlea femur & Lateral region patella & Lateral trochlea femur & & \\
\hline & & & Medial trochlea femur & & & \\
\hline & & & Lateral trochlea femur & & & \\
\hline & \multirow[t]{5}{*}{ Other } & - & - & Subspinous region tibia & - & $\begin{array}{l}\text { Hoffa's fat pad } \\
\text { (infrapatellar) }\end{array}$ \\
\hline & & & & & & $\begin{array}{l}\text { Medial } \\
\text { posterior- } \\
\text { condylar region }\end{array}$ \\
\hline & & & & & & $\begin{array}{l}\text { Lateral } \\
\text { posterior- } \\
\text { condylar region }\end{array}$ \\
\hline & & & & & & Medial recess \\
\hline & & & & & & Lateral recess \\
\hline \multirow[t]{4}{*}{$\begin{array}{l}\text { Score (per } \\
\text { region) }\end{array}$} & & $0=$ none & $0=$ none & $0=$ none & $\begin{array}{l}0= \\
\text { physiological } \\
\text { amount }^{\mathrm{a}}\end{array}$ & $0=a b s e n t$ \\
\hline & & $1=<10 \%$ of surface area & $1=$ mild & $1=<10 \%$ of bone volume & $1=$ small $^{\mathrm{b}}$ & $1=$ present \\
\hline & & $\begin{array}{l}2=10 \text { to } 75 \% \text { of surface } \\
\text { area }\end{array}$ & $2=$ moderate & $2=10$ to $25 \%$ of bone volume & $2=$ medium $^{c}$ & \\
\hline & & $3=>75 \%$ of surface area & $3=$ severe & $3=>25 \%$ of bone volume & $3=\operatorname{larg} \mathrm{e}^{\mathrm{d}}$ & \\
\hline Details & & & $\begin{array}{l}\text { An osteophyte must be visible } \\
\text { on two consecutive slices. } \\
\text { Largest osteophyte within } \\
\text { region scored }\end{array}$ & $\begin{array}{l}\text { If } \mathrm{BML} \text { span more than one } \\
\text { region, then full size of } \mathrm{BML} \text { is } \\
\text { attributed to region that is } \\
\text { most involved }\end{array}$ & & \\
\hline
\end{tabular}

BML, bone marrow lesions; LTF, lateral tibiofemoral compartment; MTF, medial tibiofemoral compartment; PF, patellafemoral compartment. ${ }^{\mathrm{a}}$ In supra-patellar bursa only. ${ }^{b}$ Fluid continuous in retropatellar space. 'With slight convexity of suprapatellar bursa. ${ }^{\mathrm{d}}$ Evidence of capsular distention.

\section{Results}

From a total of 112 potential candidates that participated in a randomized controlled trial [34] from January 2010, seven persons were excluded (because MRI could not be scheduled before the start of the trial). Patient characteristics of the study sample $(n=105)$ are presented in Table 2. In general, study participants demonstrated multiple severe tissue abnormalities, detected by both CR and MRI, indicating an advanced OA group. An overview of all study findings is presented in Table 3.
One feature from CR (JSW in the PF compartment) was found to be significantly associated with lower quadriceps strength $(\beta=-0.18, P=0.030)$, as shown in Table 4 . Three CR features were related to varus-valgus laxity; namely, JSW ( $\beta=0.26, P=0.004)$ and osteophytes $(\beta=0.26, P=$ $0.005)$ in the LTF compartment related to higher laxity, and JSW in the MTF compartment related to lower laxity $(\beta=-0.22, P=0.016)$. Three MRI features were significantly associated with lower quadriceps strength - namely, cartilage integrity (that is, reduced cartilage thickness) in 
Table 2 Descriptive data of study population $(n=105)$

\begin{tabular}{|c|c|c|}
\hline & Mean \pm SD & $n(\%)$ \\
\hline \multicolumn{3}{|l|}{ Demographics } \\
\hline Age (years) & $61.4 \pm 6.9$ & \\
\hline Gender (female) & & $73(70)$ \\
\hline Duration of knee complaints (years) & $11.3 \pm 9.3$ & \\
\hline Body mass index $\left(\mathrm{kg} / \mathrm{m}^{2}\right)$ & $29.1 \pm 4.7$ & \\
\hline \multicolumn{3}{|l|}{ Radiographic severity $^{a}$} \\
\hline K-L score $0 / 1$ & & $32(31)$ \\
\hline K-L score 2 & & $28(27)$ \\
\hline K-L score 3 & & $26(25)$ \\
\hline K-L score 4 & & $19(18)$ \\
\hline Pain severity (NRS, 0 to 10 ) & $5.1 \pm 2.1$ & \\
\hline \multicolumn{3}{|l|}{ Biomechanical factors } \\
\hline Quadriceps muscle strength $(\mathrm{Nm} / \mathrm{kg})^{\mathrm{a}}$ & $0.89 \pm 0.47$ & \\
\hline Hamstrings muscle strength $(\mathrm{Nm} / \mathrm{kg})^{a}$ & $0.61 \pm 0.26$ & \\
\hline Proprioceptive accuracy (degrees) ${ }^{a}$ & $2.9 \pm 1.9$ & \\
\hline Varus-valgus laxity (degrees) ${ }^{a}$ & $6.9 \pm 2.8$ & \\
\hline \multicolumn{3}{|l|}{ Physical examination findings } \\
\hline Bony tenderness (yes) ${ }^{\mathrm{a}}$ & & $75(71)$ \\
\hline Crepitus (yes) $^{a}$ & & $86(82)$ \\
\hline Bony enlargement (yes) ${ }^{a}$ & & $12(11)$ \\
\hline Palpable warmth (yes) ${ }^{a}$ & & $4(4)$ \\
\hline Tissue abnormalities, detected by CR & $\begin{array}{l}\text { Median } \\
\text { (range) }\end{array}$ & $n$ \\
\hline \multicolumn{3}{|l|}{ Joint space width ${ }^{a}$} \\
\hline $\operatorname{MTF}(0,1,2,3)^{b}$ & & $5,29,31,40$ \\
\hline $\operatorname{LTF}(0,1,2,3)^{\mathrm{b}}$ & & $68,20,10,7$ \\
\hline $\mathrm{PF}(0,1,2,3)^{\mathrm{b}}$ & & $31,44,22,8$ \\
\hline \multicolumn{3}{|l|}{ Osteophytes ${ }^{\mathrm{a}}$} \\
\hline $\operatorname{MTF}(0,1,2,3)^{c}$ & & $21,57,24,3$ \\
\hline $\operatorname{LTF}(0,1,2,3)^{c}$ & & $43,49,11,2$ \\
\hline $\operatorname{PF}(0,1,2,3)^{c}$ & & $11,67,25,2$ \\
\hline \multicolumn{3}{|l|}{$\begin{array}{l}\text { Tissue abnormalities, detected by } \\
\text { MRI }\end{array}$} \\
\hline \multicolumn{3}{|l|}{ Cartilage integrity ${ }^{\mathrm{a}}$} \\
\hline MTF (0 to 6) & $4(0$ to 6$)$ & \\
\hline $\operatorname{LTF}(0$ to 6$)$ & $2(0$ to 6$)$ & \\
\hline $\mathrm{PF}(0$ to 12$)$ & $2(0$ to 12$)$ & \\
\hline \multicolumn{3}{|l|}{ Osteophytes $^{\mathrm{a}}$} \\
\hline MTF (0 to 9) & $3(0$ to 9$)$ & \\
\hline LTF (0 to 9) & $2(0$ to 9$)$ & \\
\hline $\mathrm{PF}(0$ to 18$)$ & 5 (0 to 12$)$ & \\
\hline \multicolumn{3}{|l|}{ Bone marrow lesions ${ }^{a}$} \\
\hline MTF (0 to 6) & $1(0$ to 6$)$ & \\
\hline $\operatorname{LTF}(0$ to 6$)$ & $0(0$ to 6$)$ & \\
\hline $\mathrm{PF}(0$ to 12$)$ & $0(0$ to 8$)$ & \\
\hline Effusion $(0,1,2,3)^{\mathrm{a}, \mathrm{d}}$ & & $\begin{array}{l}34,31,27 \\
13\end{array}$ \\
\hline \multicolumn{3}{|l|}{ Synovitis ${ }^{a}$ presence, $n(\%)$} \\
\hline In at least one region & & $36(34)$ \\
\hline In Hoffa's fat pad & & $31(30)$ \\
\hline In medial posterior-condylar region & & $13(12)$ \\
\hline
\end{tabular}

Table 2 Descriptive data of study population $(n=105)$ (Continued)

\begin{tabular}{lc} 
In lateral posterior-condylar region & $12(11)$ \\
In medial recess & $6(6)$ \\
In lateral recess & $7(7)$ \\
\hline R, conventional radiography; K-L, Kellgren-Lawrence; LTF, lateral tibiofemoral \\
compartment; MRI, magnetic resonance imaging; MTF, medial tibiofemoral \\
compartment; NRS: numeric rating scale; PF, patellafemoral compartment; SD, \\
standard deviation. ${ }^{a}$ Data from index knee. ${ }^{\mathrm{b}} 0=$ none, $1=$ minute narrowing (0 \\
$33 \%) ; 2=$ definite narrowing (33 to $66 \%) ; 3=$ ankylosis (66 to $99 \%) .{ }^{\mathrm{C}} 0=$ none, \\
$=$ minute, $2=$ definite, $3=$ large. ${ }^{\mathrm{d}} 0=$ none, $1=$ mild; $2=$ moderate; $3=$ severe.
\end{tabular}

the PF compartment $(\beta=-0.17, P=0.041)$ (see Figure 1 ), effusion $(\beta=-0.16, P=0.049)$ and synovitis $(\beta=-0.21, P=$ 0.011 ) (see Figure 2) - while a borderline significant association was found for MTF cartilage integrity $(\beta=-0.15, P=$ 0.073). We also found an association between MRIdetected cartilage integrity in the MTF compartment and laxity $(\beta=-0.22, P=0.017)$.

No associations were found between features from CR and findings from physical examination, as shown in Table 5. On the contrary, multiple MRI features (that is, LTF cartilage integrity, osteophytes in MTF, LTF and PF compartments and effusion) were found to be significantly associated with crepitus, while the association with MTF cartilage integrity was of borderline significance $(P=0.050)$.

\section{Discussion}

This is the first knee OA study exploring associations between multiple tissue abnormalities, biomechanical factors and physical examination findings. The study provided several clinically relevant findings. Firstly, the clinically relevant and new finding that high-resolution 3.0 Tesla MRI-detected effusion and synovitis, associated with quadriceps weakness. Secondly, several tissue abnormalities (that is, cartilage integrity, osteophytes and effusion), but only when detected by MRI, were found to be associated with the presence of crepitus. Thirdly, we found associations of cartilage integrity with quadriceps weakness and reduced varus-valgus laxity.

The present explorative study showed only a limited amount of significant associations, which indicates discordance between tissue abnormalities and clinical features in knee OA patients. The lack of significant associations between radiographic and clinical features is not surprising, as the discordance between radiographic and clinical OA [13] is well known and, at least partly, related to the heterogeneity of OA. However, since pain severity has been found to be more closely linked to MRI features than to CR features [47], we were surprised by the limited amount of significant associations between MRI features, biomechanical factors and physical examination findings. 
Table 3 Summary of study findings (significant associations*)

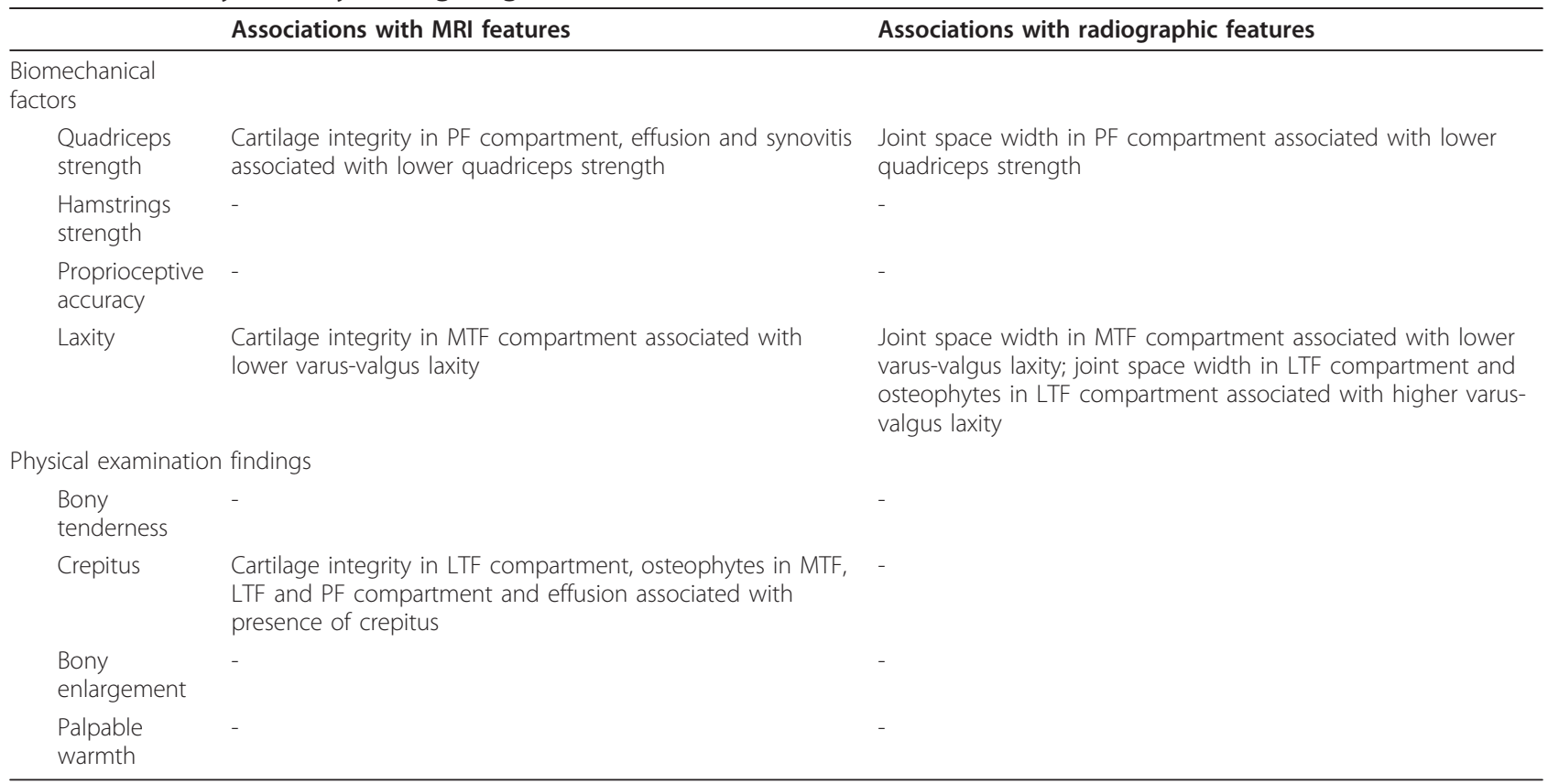

LTF, lateral tibiofemoral compartment; MRI, magnetic resonance imaging; MTF, medial tibiofemoral compartment; $\mathrm{PF}$, patellafemoral compartment. ${ }^{*} P<0.05$.

Table 4 Regression analyses ${ }^{a}$ of association between tissue abnormalities detected by CR and/or MRI and biomechanical factors

\begin{tabular}{|c|c|c|c|c|c|}
\hline & & $\begin{array}{l}\text { Quadriceps strength }(\mathrm{Nm} / \\
\mathrm{kg})\end{array}$ & $\begin{array}{l}\text { Hamstrings strength }(\mathrm{Nm} / \\
\mathrm{kg})\end{array}$ & $\begin{array}{l}\text { Proprioceptive accuracy } \\
\text { (degrees) }\end{array}$ & $\begin{array}{l}\text { Varus-valgus laxity } \\
\text { (degrees) }\end{array}$ \\
\hline \multicolumn{6}{|c|}{ Conventional radiography } \\
\hline \multirow[t]{3}{*}{$J S W^{b}$} & MTF & $-0.12(0.16)$ & $0.02(0.84)$ & $-0.03(0.78)$ & $-0.22(0.02)$ \\
\hline & LTF & $-0.07(0.37)$ & $-0.03(0.71)$ & $-0.08(0.42)$ & $0.26(<0.01)$ \\
\hline & PF & $-0.18(0.03)$ & $0.09(0.28)$ & $-0.03(0.80)$ & $-0.14(0.13)$ \\
\hline \multirow[t]{3}{*}{ Osteophyte $^{\mathrm{b}}$} & MTF & $-0.03(0.69)$ & $0.04(0.66)$ & $-0.10(0.31)$ & $0.07(0.47)$ \\
\hline & LTF & $-0.06(0.51)$ & $0.06(0.46)$ & $-0.05(0.63)$ & $0.26(<0.01)$ \\
\hline & $\mathrm{PF}$ & $-0.09(0.30)$ & $0.06(0.44)$ & $-0.09(0.38)$ & $-0.08(0.37)$ \\
\hline \multicolumn{6}{|c|}{ Magnetic resonance imaging } \\
\hline \multirow{3}{*}{$\begin{array}{l}\text { Cartilage } \\
\text { integrity }\end{array}$} & MTF & $-0.15(0.07)$ & $0.00(0.97)$ & $0.11(0.25)$ & $-0.22(0.02)$ \\
\hline & LTF & $-0.05(0.55)$ & $0.09(0.30)$ & $0.05(0.61)$ & $0.07(0.44)$ \\
\hline & PF & $-0.17(0.04)$ & $0.10(0.24)$ & $-0.02(0.86)$ & $-0.11(0.23)$ \\
\hline \multirow[t]{3}{*}{ Osteophytes } & MTF & $-0.05(0.56)$ & $0.11(0.18)$ & $0.08(0.43)$ & $-0.08(0.36)$ \\
\hline & LTF & $-0.08(0.33)$ & $0.05(0.58)$ & $0.02(0.88)$ & $0.02(0.87)$ \\
\hline & PF & $-0.12(0.15)$ & $-0.01(0.95)$ & $-0.03(0.77)$ & $-0.17(0.06)$ \\
\hline \multirow[t]{3}{*}{ BML } & MTF & $0.03(0.76)$ & $0.12(0.13)$ & $0.16(0.11)$ & $-0.06(0.48)$ \\
\hline & LTF & $-0.05(0.54)$ & $0.12(0.13)$ & $-0.05(0.62)$ & $0.12(0.18)$ \\
\hline & $\mathrm{PF}$ & $0.01(0.91)$ & $0.16(0.06)$ & $-0.17(0.08)$ & $-0.12(0.19)$ \\
\hline Effusion $^{c}$ & & $-0.16(0.05)$ & $-0.08(0.34)$ & $0.11(0.26)$ & $0.08(0.40)$ \\
\hline Synovitis $^{d}$ & & $-0.21(0.01)$ & $-0.11(0.17)$ & $0.03(0.76)$ & $-0.02(0.83)$ \\
\hline
\end{tabular}

Data presented as standardized regression coefficient $\beta$ ( $P$ value). Significant associations $(P<0.05)$ in bold. BML, bone marrow lesion; $C R$, conventional radiography; JSW, joint space width; LTF, lateral tibiofemoral compartment; MRI, magnetic resonance imaging; MTF, medial tibiofemoral compartment; PF: patellafemoral compartment. ${ }^{a}$ Adjusted for age, gender and duration of knee symptoms. ${ }^{b}$ Definite/severe abnormality compared with none/mild abnormality (reference group). 'Medium/large effusion compared with no/small effusion (reference group). ${ }^{\mathrm{d}}$ Presence of synovitis in at least one region compared with absence of synovitis (reference group). 


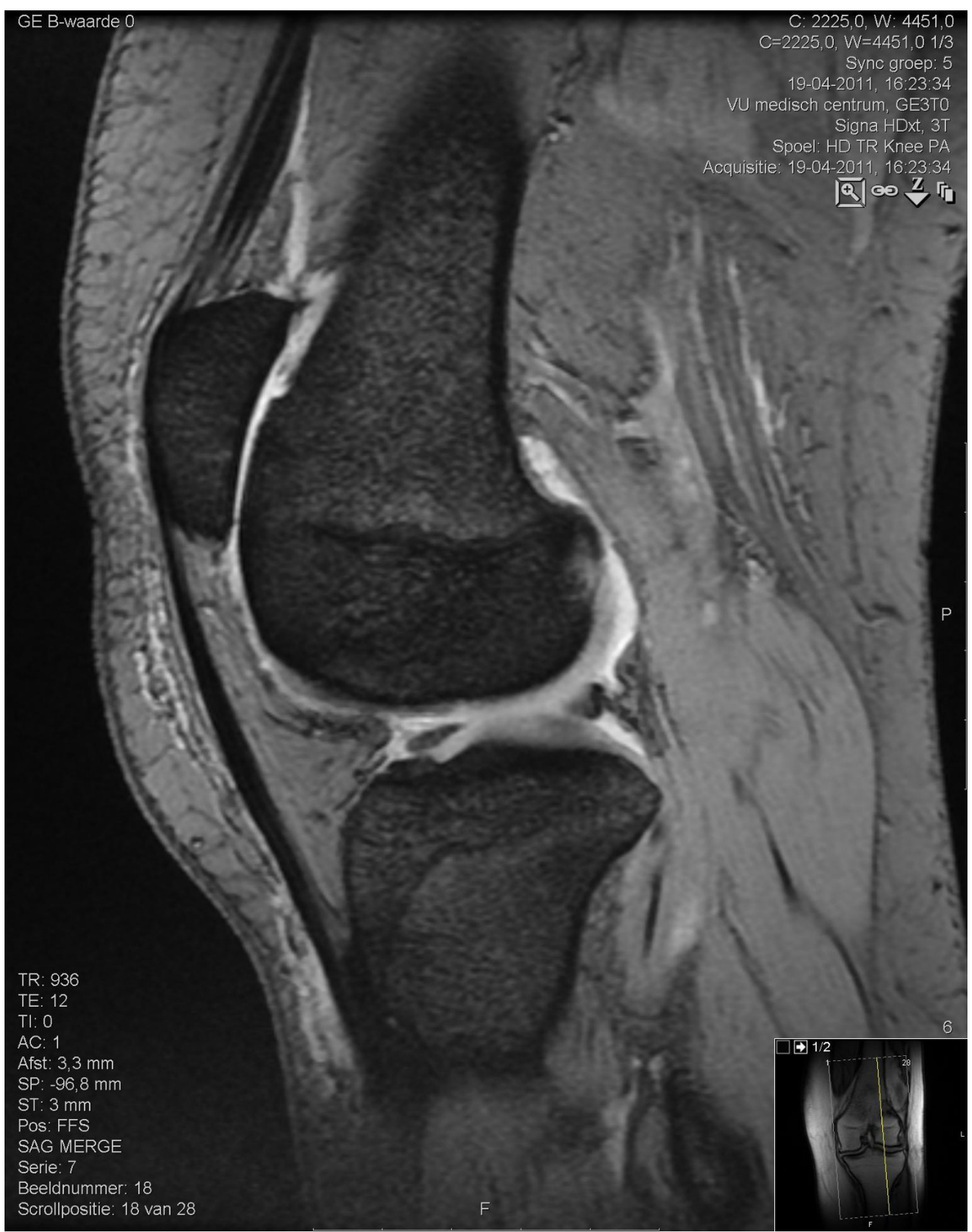

Figure 1 Reduced cartilage integrity in the patellafemoral compartment. Sagittal combined multi-echo gradient echo 3.0 Tesla magnetic resonance imaging scan.

High-resolution MRI and CR provided similar patterns of association. Firstly, reduced PF cartilage integrity, both MRI based and CR based, was associated with quadriceps weakness, which confirms previous studies $[18,20,21]$.
Secondly, MRI-based and CR-based MTF cartilage integrity loss was related to lower varus-valgus laxity. Although these associations were weak and inconsistent with previous studies $[8,22,23]$, they might be indicative for an 


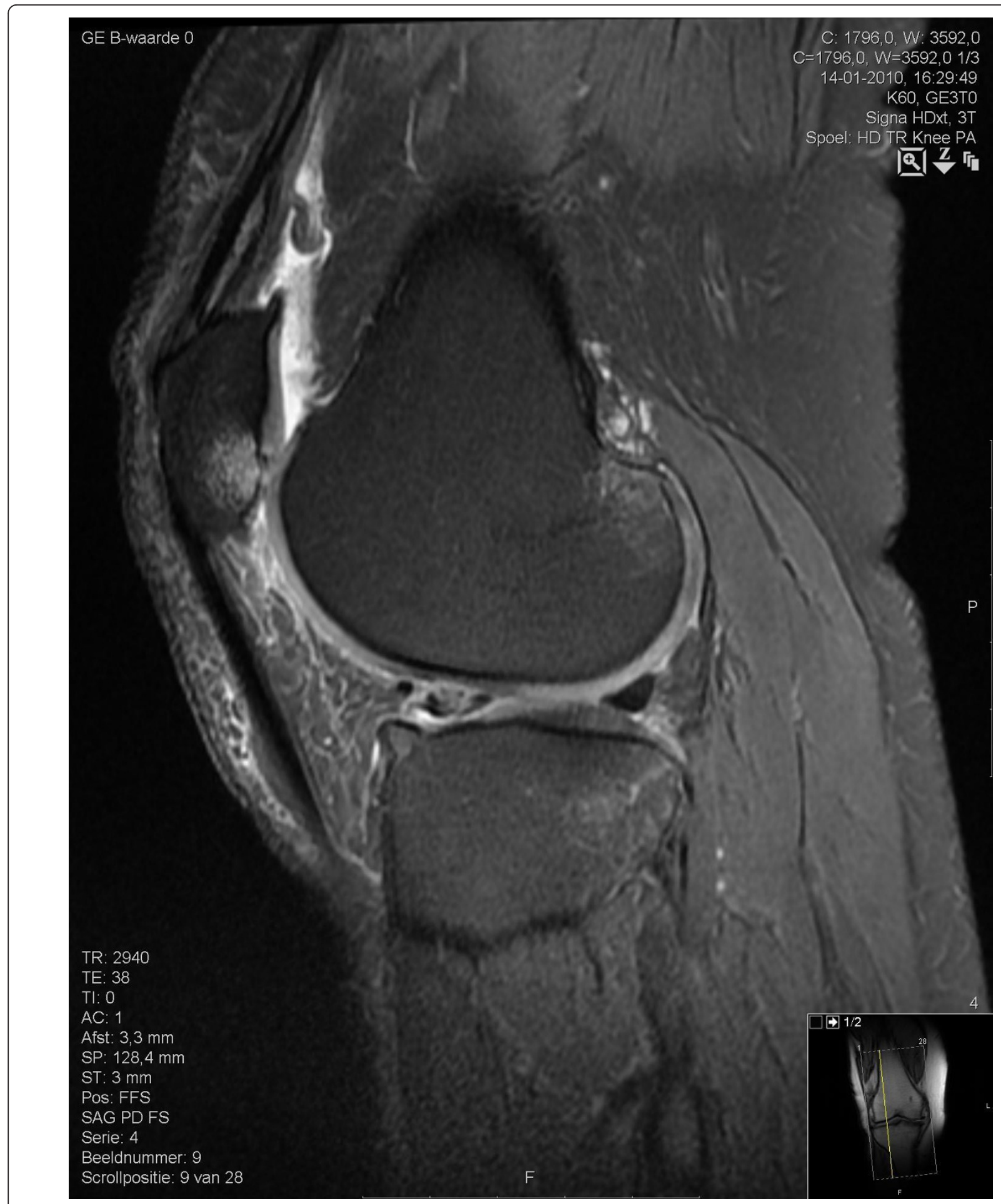

Figure 2 Infrapatellar synovitis. Fat-suppressed sagittal proton density-weighted turbo spin-echo 3.0 Tesla magnetic resonance imaging scan.

ankylosing effect of end-stage cartilage integrity (that is, reduced joint motion due to bone-to-bone) [48]. Others suggested that cartilage loss results in higher laxity due to reduced tension on ligaments (pseudo-laxity [8]), which might underlie our finding of reduced LTF cartilage integrity (but only on CR) associated with higher laxity. Future studies are needed to clarify the association between cartilage integrity and laxity. Thirdly, neither features from MRI nor from CR were significantly related to hamstrings strength and proprioceptive accuracy. Finally, 
Table 5 Regression analyses ${ }^{\mathrm{a}}$ of association between tissue abnormalities from CR and/or MRI and physical examination findings

\begin{tabular}{|c|c|c|c|c|c|}
\hline & & Bony tenderness (presence) & Crepitus (presence) & Bony enlargement (presence) & Palpable warmth (presence) \\
\hline \multicolumn{6}{|c|}{ Conventional radiography } \\
\hline \multirow[t]{3}{*}{$J S W^{b}$} & MTF & $0.90(0.82)$ & $2.13(0.15)$ & $2.22(0.34)$ & $0.44(0.43)$ \\
\hline & LTF & $0.96(0.95)$ & $4.24(0.18)$ & $1.70(0.50)$ & $\mathrm{n} / \mathrm{a}$ \\
\hline & PF & $1.80(00.27)$ & $1.15(0.82)$ & $3.09(0.09)$ & $2.72(0.35)$ \\
\hline \multirow[t]{3}{*}{ Osteophytes $^{b}$} & MTF & $0.77(0.59)$ & $1.40(0.58)$ & $0.78(0.74)$ & $0.90(0.93)$ \\
\hline & LTF & $0.85(0.81)$ & $\mathrm{n} / \mathrm{a}$ & $1.29(0.80)$ & $2.33(0.53)$ \\
\hline & PF & $1.34(0.58)$ & $1.45(0.56)$ & $1.89(0.34)$ & $3.52(0.23)$ \\
\hline \multicolumn{6}{|c|}{ Magnetic resonance imaging } \\
\hline \multirow[t]{3}{*}{ Cartilage integrity } & MTF & $0.89(0.25)$ & $1.25(0.05)$ & $1.18(0.26)$ & $0.92(0.72)$ \\
\hline & LTF & $0.88(0.27)$ & $1.61(0.02)$ & $1.26(0.15)$ & $0.75(0.38)$ \\
\hline & PF & $1.03(0.74)$ & $1.07(0.49)$ & $0.94(0.60)$ & $1.30(0.11)$ \\
\hline \multirow[t]{3}{*}{ Osteophytes } & MTF & $0.92(0.32)$ & $1.34(0.02)$ & $1.05(0.69)$ & $1.24(0.31)$ \\
\hline & LTF & $1.06(0.60)$ & $1.57(0.01)$ & $1.29(0.08)$ & $1.19(0.48)$ \\
\hline & PF & $1.09(0.20)$ & $1.20(0.04)$ & $0.99(0.90)$ & $1.30(0.17)$ \\
\hline \multirow[t]{3}{*}{$\mathrm{BML}$} & MTF & $0.97(0.76)$ & $1.16(0.27)$ & $1.01(0.94)$ & $1.04(0.87)$ \\
\hline & LTF & $0.91(0.53)$ & $1.38(0.26)$ & $1.45(0.05)$ & $1.03(0.95)$ \\
\hline & PF & $0.84(0.21)$ & $1.73(0.12)$ & $0.66(0.28)$ & $1.29(0.28)$ \\
\hline Effusion $^{c}$ & & $0.75(0.52)$ & $7.05(0.01)$ & $1.10(0.89)$ & $5.93(0.14)$ \\
\hline Synovitis $^{d}$ & & $0.84(0.70)$ & $2.14(0.21)$ & $1.30(0.70)$ & $\mathrm{n} / \mathrm{a}$ \\
\hline
\end{tabular}

Data presented as odds ratio $(P$ value). Significant associations $(P<0.05)$ in bold. BML, bone marrow lesion; $C R$, conventional radiography; JSW, joint space width; LTF, lateral tibiofemoral compartment; MRI, magnetic resonance imaging; MTF, medial tibiofemoral compartment; $n / a$, not applicable because of empty cells in

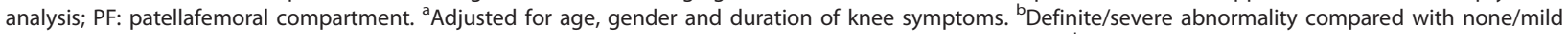
abnormality (reference group). ' $M$ Medium/large effusion compared with no/small effusion (reference group). ${ }^{\mathrm{d}}$ Presence of synovitis in at least one region compared with absence of synovitis (reference group).

no associations were found between tissue abnormalities (MRI and CR) and physical examination findings (except for crepitus), which might, at least partly, be explained by the low proportion of persons with bony enlargement $(11 \%)$ or palpable warmth $(4 \%)$ in our cohort. These similar patterns of findings from CR and MRI were determined in a study sample of patients with advanced knee OA with knee complaints for more than 10 years on average. Since MRI is able to detect tissue abnormalities at a much earlier stage of the disease than CR [31], a different pattern of associations with clinical features may possibly be found in an early OA sample. On the other hand, two results from our study may be indicative for an additional value of MRI over CR. Firstly, MRI-based effusion and synovitis, which cannot be detected by $\mathrm{CR}$, were found to be significantly associated with quadriceps weakness. Secondly, crepitus of the knee was associated with multiple MRI features (that is, LTF cartilage integrity, osteophytes in all three compartments and effusion), similar to a recently conducted population-based study [33], but was not associated with any feature from CR. This indicates that MRI seems to be able to visualize features underlying crepitus, while CR is not.

A new and potentially important finding from our study is the association of OA-related inflammation (effusion and/or synovitis) with quadriceps weakness, which is in line with previous experimental studies demonstrating an effect of effusion on quadriceps function [49-51]. Quadriceps muscles are considered the most important muscles for knee movements, stabilization and shock absorption [11]. Persons with synovitis and/or effusion had significantly lower quadriceps strength compared with persons without synovitis/effusion. In secondary analyses, similar results were yielded after adjustment for pain severity, indicating that pain does not explain the association between inflammation and quadriceps weakness. Because inflammation of the synovial membrane had mostly been identified in the infrapatellar region (that is, in $86 \%$ of persons with synovitis), which is adjacent to the patellar tendon of the quadriceps muscles, it seems plausible that quadriceps function is affected by inflammatory processes nearby the patella. In addition, inflammation may occur inside the muscle as well, which could result in decreased muscle strength [52]. Effusion is presumed to cause muscle weakness by muscle reflex inhibition due to increased intra-articular pressure [49-51]. Although knee joint inflammation has been suggested to also affect proprioceptive accuracy [7], we were not able to demonstrate this. A possible explanation could be that our study participants demonstrated relatively healthy proprioceptive accuracy. Previous studies provided conflicting results for the role of non-inflammatory effusion (that is, saline injections) in 
proprioceptive accuracy $[53,54]$. Future studies need to focus on OA-related inflammation, instead of non-inflammatory injections, to clarify the role of inflammation in proprioception.

The associations between effusion, synovitis and quadriceps weakness could be highly relevant for selecting knee OA treatment. If inflammatory processes underlie quadriceps weakness, regular quadriceps strengthening exercises are not likely to be beneficial and anti-inflammatory therapy might be needed first. This implication is in line with a recent study in which patients treated with both NSAIDs and exercises improved more in muscle strength compared with patients treated with exercises only [55]. In addition, our data revealed that physical examination of the knee strongly underestimated the prevalence of inflammation of the knee, since warmth was palpated in only $4 \%$ of our participants, compared with a prevalence of $34 \%$ for synovitis and 39\% for medium/ large effusion, detected by MRI. This implies that MRI may have additional value for clinical assessment in patients with inflammation. Our findings also emphasize that OA is not only characterized by cartilage degeneration and bony changes but also by inflammatory changes, which may point out the importance of anti-inflammatory therapies in knee OA.

Our study design has some limitations that need to be noticed. Firstly, we did not use contrast-enhanced MR imaging techniques to minimize risks for participants (for example, risk of allergic reactions, nephropathy). Because of the well-known superiority of contrastenhanced MRI for synovitis detection [56], it is remarkable that even without contrast infusions we were able to detect an association of both effusion and synovitis with quadriceps weakness. Although noncontrast-enhanced MRI demonstrated lower specificity for detecting synovitis compared with contrast-enhanced MRI, meaning that signal intensity alterations do not always represent synovitis, it is also been found to be a highly sensitive technique $(\approx 100 \%$ sensitivity) for synovitis detection [57]. This implies that the prevalence of synovitis in our study could be an overestimation, but that all persons with actual synovitis have presumably been detected. Secondly, we are not sure whether the power of our study was sufficient. Most MRI studies included large cohorts $(n>200)$, while our study consists of 105 participants. This sample size may have resulted in loss of statistical power. In addition, participants had been selected based on the presence of knee joint instability, since they participated in a study on the effectiveness of a knee stabilization exercise program, which may have introduced selection bias in the present study. Thirdly, our study design was cross-sectional with no control group, while a longitudinal design, preferably using a control group of patients at risk, is necessary to unravel interactions between tissue abnormalities, biomechanical factors and physical examination findings. However, this study has a unique design because it is the first study we are aware of in which associations could be explored between both radiography and MRI with biomechanical and physical examination features in a knee OA cohort.

\section{Conclusions}

This explorative study detected several new and clinically relevant findings, including associations of MRIbased effusion/synovitis with quadriceps weakness. Inflammation was detected in over one-third of the participants, emphasizing the inflammatory component of $\mathrm{OA}$ and a possible important role of anti-inflammatory therapies in knee OA. In general, OA-related tissue abnormalities of the knee, even those detected by MRI, were found to be discordant with biomechanical and physical examination features. As this is an explorative study, replication in future research is needed.

\section{Abbreviations}

BML: bone marrow lesions; CR: conventional radiography; JSW: joint space width; LTF: lateral tibiofemoral; MRI: magnetic resonance imaging; MTF: medial tibiofemoral; NSAID: nonsteroidal anti-inflammatory drug; OA: osteoarthritis; PF: patellafemoral.

\section{Authors' contributions}

JK, JD and WFL were responsible for conception and design. JK, JD, JPK, MvdL, MvdE, DR, REV, MG, LDR, MPMS and WFL were responsible for acquisition of data or analysis and interpretation of data. All authors were responsible for drafting the article or revising it critically for important intellectual content. All authors read and approved the manuscript for publication. JK takes full responsibility for the integrity of the work as a whole, from inception to finished article.

\section{Competing interests}

The authors declare that they have no competing interests.

\section{Acknowledgements}

The authors gratefully acknowledge T Schweigmann for obtaining MRI scans and S Romviel for biomechanical and clinical assessments. The study was funded by the Dutch Arthritis Association and Servier. The study sponsors had no involvement in the study.

\section{Author details}

${ }^{1}$ Amsterdam Rehabilitation Research Center - Reade, Dr. Jan van Breemenstraat 2, 1056 AB Amsterdam, the Netherlands. '2Department of Rehabilitation Medicine/EMGO, VU University Medical Center, De Boelelaan 1118, $1081 \mathrm{HZ}$ Amsterdam, the Netherlands. ${ }^{3}$ Department of Psychiatry, VU University Medical Center, De Boelelaan 1118, $1081 \mathrm{HZ}$ Amsterdam, the Netherlands. ${ }^{4}$ Department of Radiology, VU University Medical Center, De Boelelaan 1118, $1081 \mathrm{HZ}$ Amsterdam, the Netherlands. ${ }^{5} \mathrm{Jan}$ van Breemen Research Institute - Reade, Dr. Jan van Breemenstraat 2, 1056 AB Amsterdam, the Netherlands. ${ }^{6}$ School of Health and Life Sciences, Institute for Applied Health Research, Glasgow Caledonian University, Cowcaddens Road, Glasgow G4 OBA, UK. '7Department of Rheumatology, VU University Medical Center, VU University Medical Center, De Boelelaan 1117, 1081 HV Amsterdam, the Netherlands.

Received: 7 May 2012 Revised: 29 June 2012 Accepted: 5 October 2012 Published: 5 October 2012 


\section{References}

1. Felson DT: An update on the pathogenesis and epidemiology of osteoarthritis. Radiol Clin North Am 2004, 42:1-9, v.

2. Bijlsma JW, Berenbaum F, Lafeber FP: Osteoarthritis: an update with relevance for clinical practice. Lancet 2011, 377:2115-2126.

3. Fitzgerald GK, Piva SR, Irrgang JJ: Reports of joint instability in knee osteoarthritis: its prevalence and relationship to physical function. Arthritis Rheum 2004, 51:941-946.

4. Schmitt LC, Fitzgerald GK, Reisman AS, Rudolph KS: Instability, laxity, and physical function in patients with medial knee osteoarthritis. Phys Ther 2008, 88:1506-1516.

5. Knoop J, van der Leeden M, van der Esch M, Thorstensson CA, Gerritsen M, Voorneman RE, Lems WF, Roorda LD, Dekker J, Steultjens MP: Association of lower muscle strength with self-reported knee instability in osteoarthritis of the knee: results from the Amsterdam Osteoarthritis Cohort. Arthritis Care Res (Hoboken) 2012, 64:38-45.

6. Steultjens MP, Dekker J, van Baar ME, Oostendorp RA, Bijlsma JW: Muscle strength, pain and disability in patients with osteoarthritis. Clin Rehabil 2001, 15:331-341.

7. Knoop J, Steultjens MP, van der Leeden M, van der Esch $M$ Thorstensson CA, Roorda LD, Lems WF, Dekker J: Proprioception in knee osteoarthritis: a narrative review. Osteoarthritis Cartilage 2011, 19:381-388.

8. Sharma L, Lou C, Felson DT, Dunlop DD, Kirwan-Mellis G, Hayes KW, Weinrach D, Buchanan TS: Laxity in healthy and osteoarthritic knees. Arthritis Rheum 1999, 42:861-870.

9. Slemenda C, Heilman DK, Brandt KD, Katz BP, Mazzuca SA, Braunstein EM, Byrd D: Reduced quadriceps strength relative to body weight: a risk factor for knee osteoarthritis in women? Arthritis Rheum 1998, 41:1951-1959.

10. Sharma L, Cahue S, Song J, Hayes K, Pai YC, Dunlop D: Physical functioning over three years in knee osteoarthritis: role of psychosocial, local mechanical, and neuromuscular factors. Arthritis Rheum 2003, 48:3359-3370.

11. Bennell KL, Hunt MA, Wrigley TV, Lim BW, Hinman RS: Role of muscle in the genesis and management of knee osteoarthritis. Rheum Dis Clin North Am 2008, 34:731-754.

12. Felson DT, Gross KD, Nevitt MC, Yang M, Lane NE, Torner JC, Lewis CE, Hurley MV: The effects of impaired joint position sense on the development and progression of pain and structural damage in knee osteoarthritis. Arthritis Rheum 2009, 61:1070-1076.

13. Bedson J, Croft PR: The discordance between clinical and radiographic knee osteoarthritis: a systematic search and summary of the literature. BMC Musculoskelet Disord 2008, 9:116.

14. Guermazi A, Roemer FW, Hayashi D: Imaging of osteoarthritis: update from a radiological perspective. Curr Opin Rheumatol 2011, 23:484-491.

15. Barker K, Lamb SE, Toye F, Jackson S, Barrington S: Association between radiographic joint space narrowing, function, pain and muscle power in severe osteoarthritis of the knee. Clin Rehabil 2004, 18:793-800.

16. Baker KR, Xu L, Zhang Y, Nevitt M, Niu J, Aliabadi P, Yu W, Felson D: Quadriceps weakness and its relationship to tibiofemoral and patellofemoral knee osteoarthritis in Chinese: the Beijing osteoarthritis study. Arthritis Rheum 2004, 50:1815-1821.

17. Segal NA, Torner JC, Felson D, Niu J, Sharma L, Lewis CE, Nevitt M: Effect of thigh strength on incident radiographic and symptomatic knee osteoarthritis in a longitudinal cohort. Arthritis Rheum 2009, 61:1210-1217.

18. Palmieri-Smith RM, Thomas AC, Karvonen-Gutierrez C, Sowers MF: Isometric quadriceps strength in women with mild, moderate, and severe knee osteoarthritis. Am J Phys Med Rehabil 2010, 89:541-548.

19. Segal NA, Glass NA, Torner J, Yang M, Felson DT, Sharma L, Nevitt M, Lewis CE: Quadriceps weakness predicts risk for knee joint space narrowing in women in the MOST cohort. Osteoarthritis Cartilage 2010, 18:769-775.

20. Stefanik JJ, Guermazi A, Zhu Y, Zumwalt AC, Gross KD, Clancy M, Lynch JA, Segal NA, Lewis CE, Roemer FW, Powers CM, Felson DT: Quadriceps weakness, patella alta, and structural features of patellofemoral osteoarthritis. Arthritis Care Res (Hoboken) 2011, 63:1391-1397.

21. Amin S, Baker K, Niu J, Clancy M, Goggins J, Guermazi A, Grigoryan M, Hunter DJ, Felson DT: Quadriceps strength and the risk of cartilage loss and symptom progression in knee osteoarthritis. Arthritis Rheum 2009, 60:189-198.

22. van der Esch M, Steultjens M, Wieringa $H$, Dinant $H$, Dekker J: Structural joint changes, malalignment, and laxity in osteoarthritis of the knee. Scand J Rheumatol 2005, 34:298-301.
23. Sharma L, Eckstein F, Song J, Guermazi A, Prasad P, Kapoor D, Cahue S, Marshall M, Hudelmaier M, Dunlop D: Relationship of meniscal damage, meniscal extrusion, malalignment, and joint laxity to subsequent cartilage loss in osteoarthritic knees. Arthritis Rheum 2008, 58:1716-1726.

24. Pottenger LA, Phillips FM, Draganich LF: The effect of marginal osteophytes on reduction of varus-valgus instability in osteoarthritic knees. Arthritis Rheum 1990, 33:853-858.

25. Dieppe PA, Cushnaghan J, Shepstone L: The Bristol 'OA500' study: progression of osteoarthritis (OA) over 3 years and the relationship between clinical and radiographic changes at the knee joint. Osteoarthritis Cartilage 1997, 5:87-97.

26. Link TM, Steinbach LS, Ghosh S, Ries M, Lu Y, Lane N, Majumdar S: Osteoarthritis: MR imaging findings in different stages of disease and correlation with clinical findings. Radiology 2003, 226:373-381.

27. Torres L, Dunlop DD, Peterfy C, Guermazi A, Prasad P, Hayes KW, Song J, Cahue S, Chang A, Marshall M, Sharma L: The relationship between specific tissue lesions and pain severity in persons with knee osteoarthritis. Osteoarthritis Cartilage 2006, 14:1033-1040.

28. Kornaat PR, Bloem JL, Ceulemans RY, Riyazi N, Rosendaal FR, Nelissen RG, Carter WO, Hellio Le Graverand MP, Kloppenburg M: Osteoarthritis of the knee: association between clinical features and MR imaging findings. Radiology 2006, 239:811-817.

29. Szebenyi B, Hollander AP, Dieppe P, Quilty B, Duddy J, Clarke S, Kirwan JR: Associations between pain, function, and radiographic features in osteoarthritis of the knee. Arthritis Rheum 2006, 54:230-235.

30. Phan CM, Link TM, Blumenkrantz G, Dunn TC, Ries MD, Steinbach LS, Majumdar S: MR imaging findings in the follow-up of patients with different stages of knee osteoarthritis and the correlation with clinical symptoms. Eur Radiol 2006, 16:608-618.

31. Javaid MK, Lynch JA, Tolstykh I, Guermazi A, Roemer F, Aliabadi P, McCulloch C, Curtis J, Felson D, Lane NE, Torner J, Nevitt M: Preradiographic $\mathrm{MRI}$ findings are associated with onset of knee symptoms: the most study. Osteoarthritis Cartilage 2010, 18:323-328.

32. Sowers $M$, Karvonen-Gutierrez $C A$, Jacobson JA, Jiang $Y$, Yosef $M$ : Associations of anatomical measures from MRI with radiographically defined knee osteoarthritis score, pain, and physical functioning. J Bone Joint Surg Am 2011, 93:241-251.

33. Crema MD, Guermazi A, Sayre EC, Roemer FW, Wong H, Thorne A, Singer J, Esdaile JM, Marra MD, Kopec JA, Nicolaou S, Cibere J: The association of magnetic resonance imaging (MRI)-detected structural pathology of the knee with crepitus in a population-based cohort with knee pain: the MoDEKO study. Osteoarthritis Cartilage 2011, 19:1429-1432

34. Knoop J, Dekker J, Leeden vd M, Esch vd M, Thorstensson CA, Gerritsen M Voorneman RE, Peter W, Rooij dM, Romviel S, Lems WF, Roorda LD, Steultjens MPM: Knee joint stabilization therapy in patients with osteoarthritis of the knee: a randomized, controlled trial. 2012, Submitted.

35. Altman R, Asch E, Bloch D, Bole G, Borenstein D, Brandt K, Christy W, Cooke TD, Greenwald R, Hochberg M: Development of criteria for the classification and reporting of osteoarthritis. Classification of osteoarthritis of the knee. Diagnostic and Therapeutic Criteria Committee of the American Rheumatism Association. Arthritis Rheum 1986, 29:1039-1049.

36. van der Esch M, Steultjens M, Harlaar J, Knol D, Lems W, Dekker J: Joint proprioception, muscle strength, and functional ability in patients with osteoarthritis of the knee. Arthritis Rheum 2007, 57:787-793.

37. van der Esch M, Steultjens M, Knol DL, Dinant H, Dekker J: Joint laxity and the relationship between muscle strength and functional ability in patients with osteoarthritis of the knee. Arthritis Rheum 2006, 55:953-959.

38. Buckland-Wright JC, Wolfe F, Ward RJ, Flowers N, Hayne C: Substantial superiority of semiflexed (MTP) views in knee osteoarthritis: a comparative radiographic study, without fluoroscopy, of standing extended, semiflexed (MTP), and schuss views. J Rheumatol 1999, 26:2664-2674.

39. Chaisson CE, Gale DR, Gale E, Kazis L, Skinner K, Felson DT: Detecting radiographic knee osteoarthritis: what combination of views is optimal? Rheumatology (Oxford) 2000, 39:1218-1221.

40. Altman RD, Gold GE: Atlas of individual radiographic features in osteoarthritis, revised. Osteoarthritis Cartilage 2007, 15(Suppl A):A1-A56.

41. Kellgren $\mathrm{JH}$, Lawrence JS: Radiological assessment of osteo-arthrosis. Ann Rheum Dis 1957, 16:494-502. 
42. Hunter DJ, Lo GH, Gale D, Grainger AJ, Guermazi A, Conaghan PG: The reliability of a new scoring system for knee osteoarthritis MRI and the validity of bone marrow lesion assessment: BLOKS (Boston Leeds Osteoarthritis Knee Score). Ann Rheum Dis 2008, 67:206-211.

43. Felson DT, Niu J, Guermazi A, Roemer F, Aliabadi P, Clancy M, Torner J, Lewis CE, Nevitt MC: Correlation of the development of knee pain with enlarging bone marrow lesions on magnetic resonance imaging. Arthritis Rheum 2007, 56:2986-2992.

44. Wildi LM, Raynauld JP, Martel-Pelletier J, Abram F, Dorais M, Pelletier JP. Relationship between bone marrow lesions, cartilage loss and pain in knee osteoarthritis: results from a randomised controlled clinical trial using MRI. Ann Rheum Dis 2010, 69:2118-2124.

45. Zhang $Y$, Nevitt M, Niu J, Lewis C, Torner J, Guermazi A, Roemer F, McCulloch C, Felson DT: Fluctuation of knee pain and changes in bone marrow lesions, effusions, and synovitis on magnetic resonance imaging. Arthritis Rheum 2011, 63:691-699.

46. Roemer FW, Guermazi A, Felson DT, Niu J, Nevitt MC, Crema MD, Lynch JA, Lewis CE, Torner J, Zhang Y: Presence of MRI-detected joint effusion and synovitis increases the risk of cartilage loss in knees without osteoarthritis at 30-month follow-up: the MOST study. Ann Rheum Dis 2011, 70:1804-1809.

47. Yusuf E, Kortekaas MC, Watt I, Huizinga TW, Kloppenburg M: Do knee abnormalities visualised on MRI explain knee pain in knee osteoarthritis? A systematic review. Ann Rheum Dis 2011, 70:60-67.

48. Brage ME, Draganich LF, Pottenger LA, Curran JJ: Knee laxity in symptomatic osteoarthritis. Clin Orthop Relat Res 1994, 304:184-189.

49. Spencer JD, Hayes KC, Alexander IJ: Knee joint effusion and quadriceps reflex inhibition in man. Arch Phys Med Rehabil 1984, 65:171-177.

50. Jones DW, Jones DA, Newham DJ: Chronic knee effusion and aspiration: the effect on quadriceps inhibition. Br J Rheumatol 1987, 26:370-374

51. Jensen K, Graf BK: The effects of knee effusion on quadriceps strength and knee intraarticular pressure. Arthroscopy 1993, 9:52-56.

52. Levinger I, Levinger P, Trenerry MK, Feller JA, Bartlett JR, Bergman N, McKenna MJ, Cameron-Smith D: Increased inflammatory cytokine expression in the vastus lateralis of patients with knee osteoarthritis. Arthritis Rheum 2011, 63:1343-1348.

53. Cho YR, Hong BY, Lim SH, Kim HW, Ko YJ, Im SA, Lee Jl: Effects of joint effusion on proprioception in patients with knee osteoarthritis: a singleblind, randomized controlled clinical trial. Osteoarthritis Cartilage 2011, 19:22-28.

54. McNair PJ, Marshall RN, Maguire K, Brown C: Knee joint effusion and proprioception. Arch Phys Med Rehabil 1995, 76:566-568.

55. Petersen SG, Beyer N, Hansen M, Holm L, Aagaard P, Mackey AL, Kjaer M: Nonsteroidal anti-inflammatory drug or glucosamine reduced pain and improved muscle strength with resistance training in a randomized controlled trial of knee osteoarthritis patients. Arch Phys Med Rehabil 2011, 92:1185-1193.

56. Crema MD, Roemer FW, Marra MD, Guermazi A: Magnetic resonance imaging assessment of subchondral bone and soft tissues in knee osteoarthritis. Rheum Dis Clin North Am 2009, 35:557-577.

57. Roemer FW, Guermazi A, Zhang Y, Yang M, Hunter DJ, Crema MD, Bohndorf K: Hoffa's fat pad: evaluation on unenhanced MR images as a measure of patellofemoral synovitis in osteoarthritis. Am J Roentgenol 2009, 192:1696-1700.

doi:10.1186/ar4050

Cite this article as: Knoop et al:: Biomechanical factors and physical examination findings in osteoarthritis of the knee: associations with tissue abnormalities assessed by conventional radiography and highresolution 3.0 Tesla magnetic resonance imaging. Arthritis Research \& Therapy 2012 14:R212.

\section{Submit your next manuscript to BioMed Central and take full advantage of:}

- Convenient online submission

- Thorough peer review

- No space constraints or color figure charges

- Immediate publication on acceptance

- Inclusion in PubMed, CAS, Scopus and Google Scholar

- Research which is freely available for redistribution

Submit your manuscript at www.biomedcentral.com/submit
Biomed Central 